\title{
UWB Radar Technology
}

\author{
Imran Ahmad $^{1}$, A.S. Hussaini ${ }^{1,2,3}$, I.T.E. Elfergani ${ }^{3}$, Ahmed S.I. Amar ${ }^{1,4}$, J. Rodriguez ${ }^{3,5}$, R.A. \\ Abd-Alhameed ${ }^{2}$ \\ \{iahmad3@bradford.ac.uk¹, ash.hussaini@aun.edu.ng², i.t.e.elfergani@av.it.pt, \\ a.a.s.ismail@bradford.ac.uk, jonathan@av.it.pt r.a.a.abd@bradford.ac.uk $\left.{ }^{2}\right\}$
}

\author{
${ }^{1}$ Bradford-Renduchintala Centre for Space AI, Faculty of Eng. \& informatics, Univ. of Bradford, BD7 \\ 1DP, UK \\ ${ }^{2}$ School of Engineering, American University of Nigeria, Yola \\ ${ }^{3}$ Instituto de Telecomunicações - Aveiro, Portugal i.t.e.elfergani, ash, jonathan \} @av.it.pt \\ ${ }^{4}$ Faculty of Engineering, Ain Shams University, Cairo, Egypt \\ ${ }^{5}$ University of South Wales, Pontypridd CF37 1DL, UK
}

\begin{abstract}
The purpose of this study is to describe how Ultra Wideband (UWB) radars differ from regular narrow band radars. The aspects described in the paper are the creation, radiation, and processing of Ultra Wideband radar signals elicited by changes in the signal waveform during the localization operation, as well as the appearance of mutual dependency between the signal waveform and antenna directivity. The receipt of the target radio image has been depicted. The major advantages of UWB radar that have been discussed in this paper are large bandwidth, carrier less signal and ultra-short width of the pulse.
\end{abstract}

Keywords: UWB Radar Technology, UWB, Ultra Wideband Radars, Features of Ultra Wideband Radars, Advantages of UWB, Disadvantages of UWB, Target Selection in UWB Radar of RFID, Detection of Target in UWB Radar.

\section{Introduction}

For transmitting information, the vast majority of existing radio systems use a restricted frequency array and moving waveforms that use harmonic (sinusoidal) or related quasiharmonic signals. The explanation for this is simple: the RLC vibration scheme, which is a simple electrical vibratory system, generates sinusoidal vibrations [1]. Ultra-Wideband radar depends upon a short pulse duration methodology and is utilized for transmitting the signal at high bandwidth frequency that is, ranging from 3.1 to $10.6 \mathrm{GHz}$. Thus, the power of this radar is very low per MHz, that is; $-41.3 \frac{\mathrm{dBm}}{\mathrm{MHz}}$

The significant properties of the current radio system consent to pick signals relying on their densities. Thus, the density array of the majority of the radio manufacturing systems is very less as compared to the broadcasted density they utilize. Both the assumption and rehearse of the current radio systems takes this offbeat attribute into contemplation. At the same time, the contracted density array of the signal diminishes the explanatory power of the radio scheme. It is for that reason, it is necessary to increase the density array for boosting the data space [2]. The only alternate is enlarging the communication time. This complexity is particularly vital for radar, when the disclosure time is continually severely incomplete [3].

The general radars with the occurrence array having not more than $10 \%$ of the shipping occurrence have almost worn out their informational aptitude. As a result, radar deception in 
service of signals with regularity arrays up to $1 \mathrm{GHz}$ (the duration of the radiated pulses approximately $1 \mathrm{~ns}$ ) has increased [4]. Due to the range devaluation of the pulse dimensions of radar, the informational content in the UWB position grows. As a result, when a sounding pulse's dimension changes from $1 \mathrm{~s}$ to $1 \mathrm{~ns}$, the pulse's power decreases from $300 \mathrm{~m}$ to $30 \mathrm{~cm} \mathrm{[5].}$

It can be assumed that the appliance that examines the liberty, becomes extra fine and delicate. It permits the attainment of the radio image of the object.

The bandwidth of Ultra-Wideband radar is extremely high approximately possessing a $500 \mathrm{MHz}$ range, fractional bandwidth $(B W)$ higher than $20 \%$ of the center frequency $\left(f_{c}\right)$. Large Absolute Bandwidth $(B W)$ is a range of frequency on either side to the central frequency. The power of each side of the band is $10 \mathrm{~dB}$. The upper-frequency band is depicted by $f_{H}$ and the lower frequency band is depicted by $f_{L}$ and bandwidth is represented below:

$$
B W=f_{H}-f_{L}
$$

The formula for central frequency is given below:

$$
f_{c}=\frac{1}{2}\left(f_{H}+f_{L}\right)
$$

The formula of the fractional bandwidth [6] is as follows:

$$
B_{f}=\frac{B W}{f_{c}}=\frac{f_{H}-f_{L}}{\frac{1}{2}\left(f_{H}+f_{L}\right)}=\frac{2\left(f_{H}-f_{L}\right)}{\left(f_{H}+f_{L}\right)} \times 100 \%
$$

Downrange resolution refers to detection on the vertical direction, referee $\Delta R$ has a direct (directly proportional) relationship with the speed wave $v(\mathrm{~m} / \mathrm{s})$ and it has an inverse relationship with the bandwidth wave $B(\mathrm{~Hz})$ which is measured in meters $(\mathrm{m})$. It is given below:

$$
\Delta \mathrm{R}=\frac{v}{2 B}
$$

It can differentiate and make the detection of an object in the horizontal direction by utilization of the Cross-range resolution $\Delta C R$. It relies on the beam width of an antenna and is determined by the antenna aperture size, which is given below:

$$
\Delta C R=\theta R
$$

Ultra Wideband (UWB) RADAR systems discharge signals over a broad range of frequency which is amid $3 \mathrm{GHz}$ to $10 \mathrm{GHz}$ as compared to the conventional RADAR systems and remain extremely difficult to be detected. The transmitted signal is vital to a very low power spectrum. The most common and best solution to generate Ultra Wideband (UWB) transmitted signal is to generate pulses with a very short time duration, generally known as PRF which are usually less than a Nano-second. Ultra-wideband radar systems utilize the transmitter antenna for transmitting the input signal possessing a very low duration of pulse mostly in nanoseconds. This input signal coming from the input transmitter antenna travels at the speed of light to be received at the receiver side. At the receiver signal, the signal is scattered in all directions. To calculate the range of the signal propagation time algorithm is used as the UWB signal travels at the speed of light [7]. Based on this range calculation, there are two kinds of Ultra Wideband radar system which are long-range and short-range Ultra Wideband radar system. The implementation of short-range radar is at the industrial level as it has the capability of detecting the signal of centimeters up to meters and provides high regulation with maximum precision 
[8]. Whereas, long-range Ultra Wideband radar systems are used for surveillance purposes frequently in the Synthetic Aperture Radar.

\section{Major Possibilities of Ultra Wideband (UWB) Radars}

The decline of the gesture extent in the Ultra Wideband (UWB) radar permits for the following major possibilities:

\subsection{Advanced Detected Object Array Dimension}

One of the major possibilities of the decline of the gesture extent in the Ultra Wideband (UWB) radar is the advanced detected object array dimension efficiency [9]. This result is an increase in radar resolution for all coordinates, although target motion by one coordinate does not need target settling by other coordinates.

\subsection{Recognition of Object Class and Sort}

Another important potential is object class and sort identification, because the received gesture contains information not only about the item as a whole, but also about its divide aspect [10].

\subsection{Reduction in the Radar Possessions of Reflexive Obstruction}

Another major possibility is the decrease in the radar possessions of reflexive obstruction from rain, mist, aerosols, metalized strips, etc. [11]. This is because the diffusion cross-section of obstruction source within a small pulse level is compact close to the object scattering the cross-section.

\subsection{Improved Cohesion Observing Point}

A main possibility of the decrease of the gesture extent in the Ultra Wideband radar is the enhanced cohesion observing point at low ascent edge at the liability of excluding the intrusion gaps in the wire arrangement [12]. This is because the main signal and any landscape return gesture appear at the wire at specific times, which facilitates their choice.

\subsection{Development of the Feasibility of Object Detection}

Another major possibility is the advancement of the feasibility of object detection and enhanced balance observing, a point at the liability of lobe format rejection, the trivial radiation mould of irradiated targets because oscillations reflected from the entity parts of the goal do not hinder and abandon, providing a more identical radar cross segment [13].

\subsection{Exemption of the Radar to the Outside Narrowband}

The development of the radar's exemption from outside narrowband electromagnetic emission possessions and commotion is another key option for reducing the gesture range in the UWB radar [14].

\subsection{Amplifying the Furtiveness of Radar}

Another major possibility is the amplification of the furtiveness of the radar by a gesture, which will be tough to identify. The above-mentioned rewards are possibly achievable [15]. Their functioning requires an abstract pedestal permitting for the estimation of the characteristics of 
UWB radars. The motive has to do with the important distinctions of the procedure of especially wideband annotations from the related procedure when ordinary narrow-band signals are used.

\section{Major Characteristics of UWB Radars}

The main characteristics of Ultra Wideband (UWB) Radars are the change in signal waveform during detection and range, as well as the antenna pattern's dependence on the signal duration and waveform. These are described in detail below:

\subsection{Alteration in the Signal Waveform in the Procedure of Detection and Ranging}

Signals in the narrow band - sinusoidal and quasi-sinusoidal - have unique properties. The shape (waveform) of sinusoidal and quasi-sinusoidal signals remains unchanged while using common gesture conversions like addition, subtraction, isolation, and combination; the signals have the same shape as the creative role and can only change in amplitude and time move [16]. The shape is defined as the law of variation of a signal in time from here on out. It changes not only the parameters but also the shape on the divergence, at the ultra-wideband gesture at certain conversions.

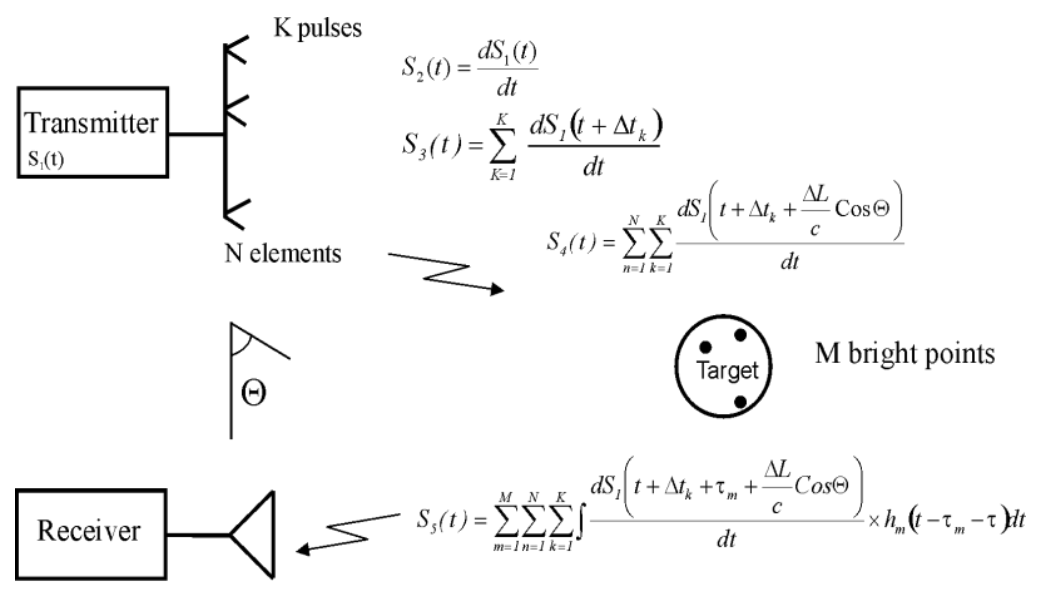

Fig. 1: The signal process.

The Ultra Wideband (UWB) signal S1 (Fig.1) is thought to be created and sent to the aerial as a current pulse. The linear size of the warmer $\mathrm{L}$ is less than the pulse period in space $\mathrm{c}$ (c is light speed, and is pulse period in time domain). Because the strength of radiated electromagnetic ground increases proportionally with the imitative (first or higher) of the aerial current, the first modification of the Ultra Wideband (UWB) signal shape (S2 in Figure 1) occurs during pulse emission.

When the aerial is agitated in one spot and modern pulses progress along the warmer [17], the contour transforms next. In this situation, the aerial rudiments with a span of $\Delta \mathrm{L}=\mathrm{c}$ will emit a series of electromagnetic gesture pulses. As a result, a single pulse becomes a series of $\mathrm{K}$ pulses separated by time intervals $\Delta \mathrm{t}$. (S3 in Figure 1). The length of the apparent warmer varies 
depending on the angle between the ordinary and the aerial range, as well as the orientation towards the point of acceptance. As a result, inter-pulse intervals differ depending on the angle:

$$
\text { Inter Pulse }==\Delta \mathrm{t} \sin (\Theta)
$$

The third change in the figure is caused by the obstruction of fields in space, which are radiated by $\mathrm{N}$ rudiments of the aerial. When compared to the pulse radiated by the adjacent antenna element, the pulse radiated by one aerial aspect at the angle $\Theta$ is delayed by the time $(\mathrm{d} / \mathrm{c}) \cos \Theta$. In comparison to the pulse emitted by the closest aerial aspect, the pooled pulse will have a variety of shapes and sizes at various angles $\Theta i n$ the far ground (S4 in Fig. 1).

The goal disperses this UWB gesture. As a result, it changes shape for the fourth time (S5 in Figure 1). M local dispersal rudiments ("bright points") are put alongside the line Lt. for the UWB signal $\mathrm{c}<<\mathrm{Lt}$. The pulse progression is formed by a UWB gesture that reflects separate target rudiments. The number of pulses, the time delay hm, and the power are all affected by the object form and aspect pulse response hm.

"Target image" is the name given to this pulse sequence. The entire image depicts the time allocation of sprinkled energy, which is achieved by using a time distance of toto $=2 \mathrm{Lt} / \mathrm{c}$. As a result, the target RCS becomes a time-dependent magnitude (the theory of immediate RSC was introduced). The image varies as the screening angle changes. The inferior outline of the target is non-stationary and varies in this scenario. Dotted signals do not last and do not produce "nulls" in the derived outline. This ensures a steady view of the object. The frequency spectrum of some target rudiments may be outside of the UWB signal continuum. Regularity filters are examples of such rudiments, which alter the shape even more.

At the answer, the shape changes for the fifth time. The reason for this is the same as for emission: temporal differences between current pulses caused by the electromagnetic ground in aerial rudiments at varied distances from the objective. Because of variable signal attenuation in different incidence bands, the 6th transform happens through signal broadcast from side to side in the ambient.

Figure 2 shows an actual illustration of a UWB signal reflected off the target.

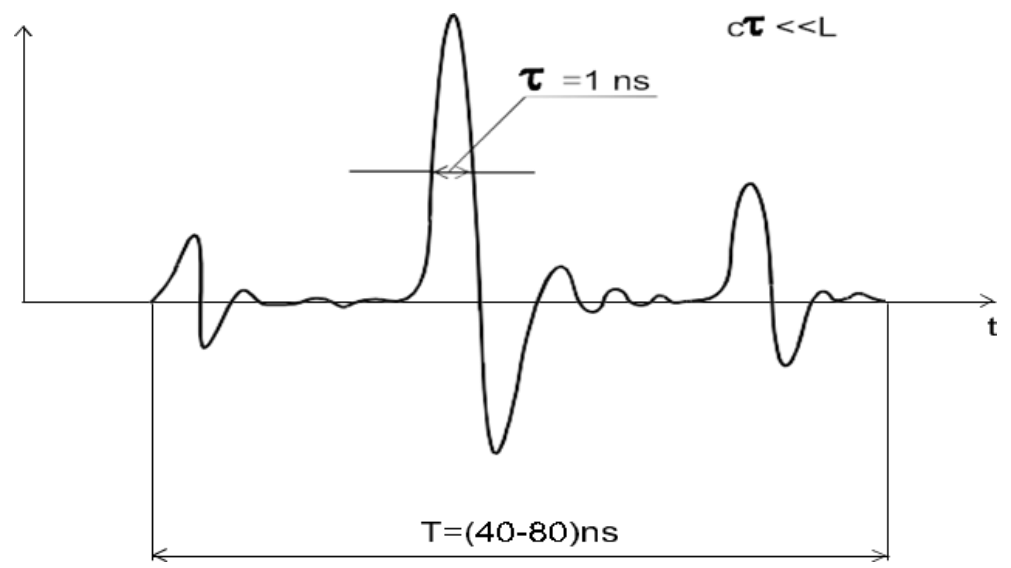




\section{Fig. 2: Target reflected signal}

\subsection{Signal Length and Waveform Differences Due to Antenna Pattern}

When the circumstance $L \operatorname{Cos} \Theta>c \tau$ is satisfied, the signal waveform begins to vary depending on the direction of radiation (reception), i.e. the space coordinates. In this scenario, there is no specific relationship between the signal amplitude and its power natural in narrowband oscillations. The construction of a typical aerial pattern based on the ground is hampered by this situation.

As a result, the aerial pattern production for UWB signals is established based on the power (Figure 3). These aerial patterns differ from the parallel aerial patterns of aerial's beam melodic and quasi-harmonic signals in important ways. There is no lobe environment in them. In order to control such an IPC mechanism, the following situation must be met.

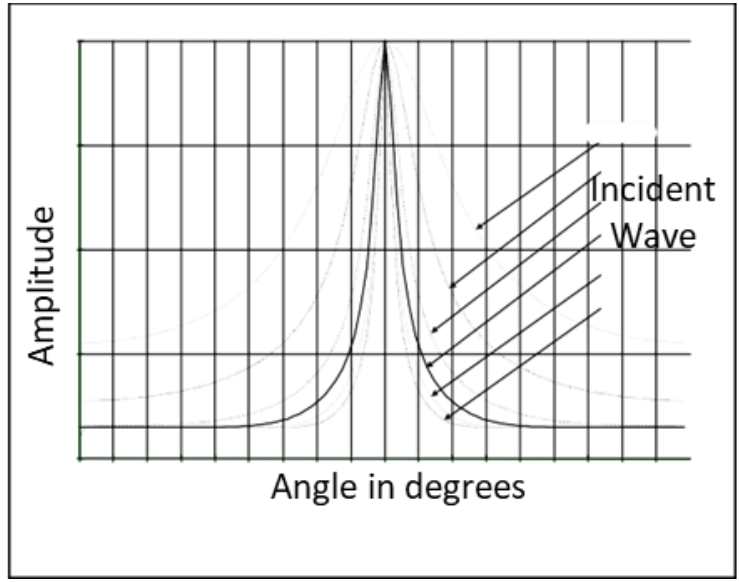

Fig. 3: Aerial Pattern Production based on the Power in UWB signals

Another difference is that adjusting the ratio between $\mathrm{c}$ and the radiator spacing $\mathrm{d}$ of an aerial array can change the measurement of the central lobe of an assortment's aerial pattern. The aerial pattern width for both the UWB signal and the narrow-band gesture is decreasing as $d$ is reduced or amplified. In contrast to the latter, however, its development does not become multi-lobe due to the lack of interference from entity radiator oscillations. This technology may theoretically be used to make the aerial pattern of a UWB signal emitting aerial as small as desired. As a result, the aerial pattern for the UWB gesture is determined not only by gaunt coordinates, but also by the time-dependent waveform S. As a result, the jargon for the UWB gesture aerial pattern will take the following shape:

$$
\mathrm{P}(\Theta, \varphi, \mathrm{S}, \mathrm{t}) \text { and } \mathrm{W}(\Theta, \varphi, \mathrm{S}, \mathrm{t})
$$

The directivity aspect $G(\Theta, S, t)$, the expand feature $K(\Theta, S, t)$, and the efficient crosssection $\mathrm{A}(,, \mathrm{S}, \mathrm{t})$ of an aerial become relying on the gesture parameters because the aerial pattern of an aerial radiating or receiving the UWB gesture gets reliant on the signal waveform and period. 


\section{Moving Target Selection in the UWB Radar}

When used under static jamming conditions, the UWB radar has an extra feature that distinguishes it from narrow-band radars [18]. Without employing the Doppler result, impacting targets can be separated with a small pulse amount. If a goal schedules a reserve beyond a sort aspect ( $30 \mathrm{~cm}$ at $\mid=1 \mathrm{~ns}$ ) above the repetition period $\mathrm{T}$, the gesture of this item will be split up and the signals of immobile or low-mobility targets will be hidden when interleaved episodic exclusion is functional.c $\tau<2 \mathrm{~V}_{\mathrm{R}} \mathrm{T}$

Where VR refers to an object's radial speed. This method of variety does not use "blind" velocities or impose specific desires on the lucidity of radiated signals. The object rate is always clearly determined. By changing the array to an object, the object radial rate VR may be resolved in the variety method. The minimum quantity that may be determined about an object's speed is: $\mathrm{V}_{\mathrm{Rmin}}=\mathrm{c}</ 2 \mathrm{~T}$

The pulse reappearance period as described above; however, the amount of conflict in flowing into and out of a small pulse number may be similar to the magnitude of interferences present at this level. This could lead to a significant de-correlation of blockage and a deterioration in the exchange-phase allowance equipment's competence. Thus, when the pulse length decreases and the radar pulse level decreases, two distinct tendencies emerge: a reduction in intrusion power and an increase in their obstruct de-correlation. Figure 4 depicts the reliance on the pulse span $\tau$ for the gesture-to-intrusion ratio Q/Q0. The notations on this diagram are as follows: $\omega_{\mathrm{d}}-$ Doppler frequency of interference

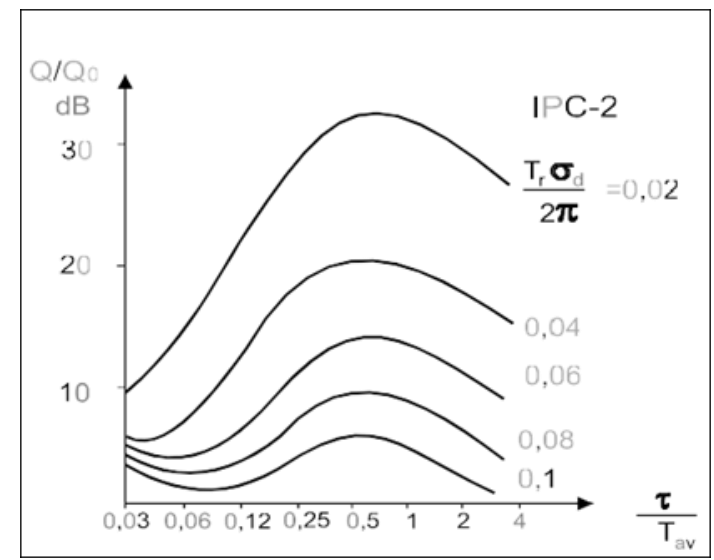

Fig. 4: Gesture-to-Intrusion Ratio

Q - signal-to-interference ratio at the alternate-period compensation output;

Q0- signal-to-interference ratio at $\tau=1$ and $\omega_{\mathbf{d}} / \mathbf{T}=0.1$.

The percentage of the normal Doppler occurrence to the pulse recurrence density $\mathrm{d} / \mathrm{T}$ is the parameter of the family of curves. The con claims that the hint-to-interference ratio rises initially due to the pulse capacity devaluation and subsequently falls due to the amplifying in the inter 
period de-correlation of noise as the pulse length contracts. The swap-period incentive system stops working completely for very small pulse spaces, and the increase in the signal-tointerference proportion is accounted for by reducing the amount of incursion inside the pulse size. Due to a stronger sensitivity of this swap-period intrusion compensation to the association features of blockage, the stipulated regularities are more clear for the more competent double swap-period wage of intrusion. As a result, in short-range, the usage of the affected target hint is appropriate for adequately slender-band interferences (e.g. local items) and a satisfactorily soaring repetition frequency.

\section{Detection of Target in the UWB Radar}

Counting the gear of signal dispersal from object intense locations, the UWB pointer alters its outline many times during the object placement method. As a result, the evolution of pulses with opening parameters is warped into a returning gesture. Because it conveys knowledge of not only object occurrence and object positions, but also object arrangement, this gesture is frequently referred to as "target image" [19]. The ability to recognise an object and generate a radio image of it is made possible by proper picture distribution. It is necessary to identify the object at an early stage of location before appreciating it. Because the arrangement of the UWB returned indicator is completely unknown, standard approaches such as finest signal dispensation by coordinated filtering or correlation with the suggestion signal are not recommended for UWB signal recognition.

It is possible to find a nameless multiunit target using normal methods. If the number of independent resolution intervals, $\mathrm{P}$, organised along a target exceeds the number of intervals, $\mathrm{Q}$, including radiant points, all combinations of $\mathrm{P}$ intervals taken $\mathrm{Q}$ bright points at a time must be accounted for in order to produce the best detector. However, this algorithm's comprehension lacks a large number of processing channels. Approaches for dispensing such messages in a quasi-optimal manner are well-known. However, when compared to optimum detectors, all quasi-optimal detectors suffer significant damage [20]. As a result, extending an optimal detector for UWB signals disseminated by a structured aim is critical.

The only known parameter of such signals is the replication period $\mathrm{T}$. This constraint could be used to create the best detector for UWB signals. It employs an inward signal as the mentioned gesture in the bordering repetition era, and it is deferred at the time spell T. As a result, the accepted signal is compared to the precise imitation signal rather than the specified signal as in a common correlation. In this situation, the external noise in two repetition durations that are close together is unrelated. As a result, the signal slice becomes the framework for resolving the correlation radar's ability (Figure 5).

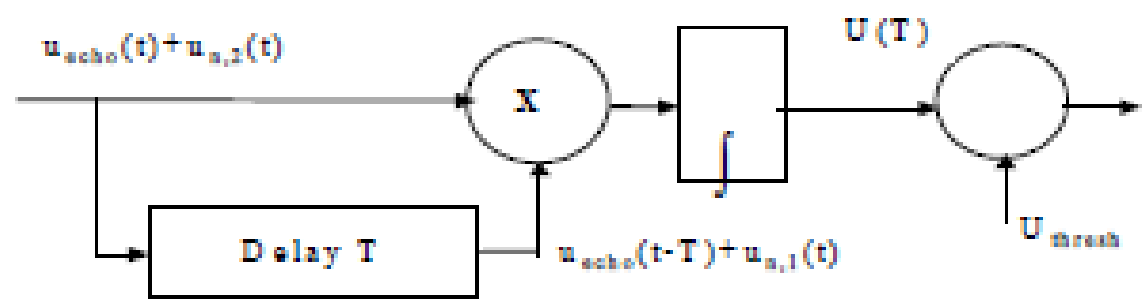

Fig. 5: Interleaved Periodic Correlation Processing (IPCP) 
Interleaved periodic correlation processing is the name given to this type of signal processing (IPCP).

a) The acknowledge signal is compared to a signal dispersed by a target, rather than a radiated signal.

b) Explosion stimulates the correlator inputs on both sides. There is a distribution activity for the output of typically distributed explosions at the Correlator outlet. c) the time of assimilation The inspection lay off, that is, the scattered signal span, deceives $\mathrm{Ti}$, not the diffuse signal extent (if a physical object range is $\mathrm{Lt}$, the integration time is equal to $\mathrm{Ti}=(2 \mathrm{Lt} / \mathrm{c})-\tau$, where is the lifetime of a radiated signal). Cogent phrases for disposal service for the device of ordinarily dispersed sounds at the IPCP outlet are really important. The graphing of the disclosure quality is done using mathematical modelling.

Figures $6 \mathrm{a}$ and $6 \mathrm{~b}$ demonstrate the IPCP disclosure quality for a signal dispersed by a stagnant target at two false alarm rates of $10^{-2}$ and $10^{-4}(\mathrm{D}$ - probability of detection, $\mathrm{q}$ - signal-to-noise ratio).
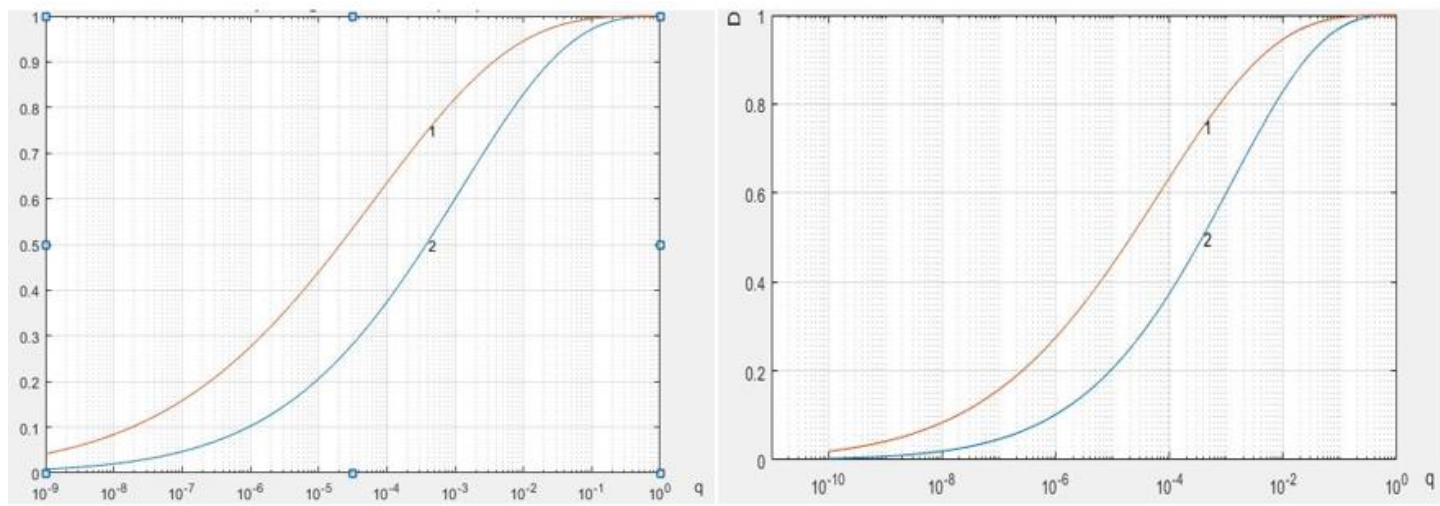

Fig. 6: Disclosure Quality of IPCP

The disclosure quality of an ordinary correlation for completely known gestures is also shown in these figures. The span of a received gesture is identical to the span of a light up gesture in order to make the comparison accurate (one point target). The results reveal that the IPCP espial personality approach, which includes the conventional correlate disclosure personality, is responsible for significant false alarm rates $\left(10^{-2}\right)$. The difference in these people's situations when it comes to lowering the number of false alarms $\left(10^{-4}\right)$. This can be explained by the extended "tails" of the disposal activities for the regularly assigned explosive product. Setting the verge level higher in IPCP than in the standard correlator allows the given level of incorrect alarm count to be maintained. At the same time, the IPCP's revealing personality is far superior to the strength radar's. The confidence of the disclosure personality on the integration time Ti is depicted in Fig. 7. (found by a target length). For modelling, the distorted alarm rate10-4 and integration times $\mathrm{Ti}$ of 2,10 , and 20 were used. This illustration also depicts the espial that distinguishes an ordinary correlator. The graphic shows that as the target length increases, the IPCP espial distinct for a static purpose approaches the standard correlator feature more and more when it exposes the entire known signal [21]. The explanation behind this is that the 
circulation purpose for the ordinarily shared explosion access the regular circulation while including the fragment.

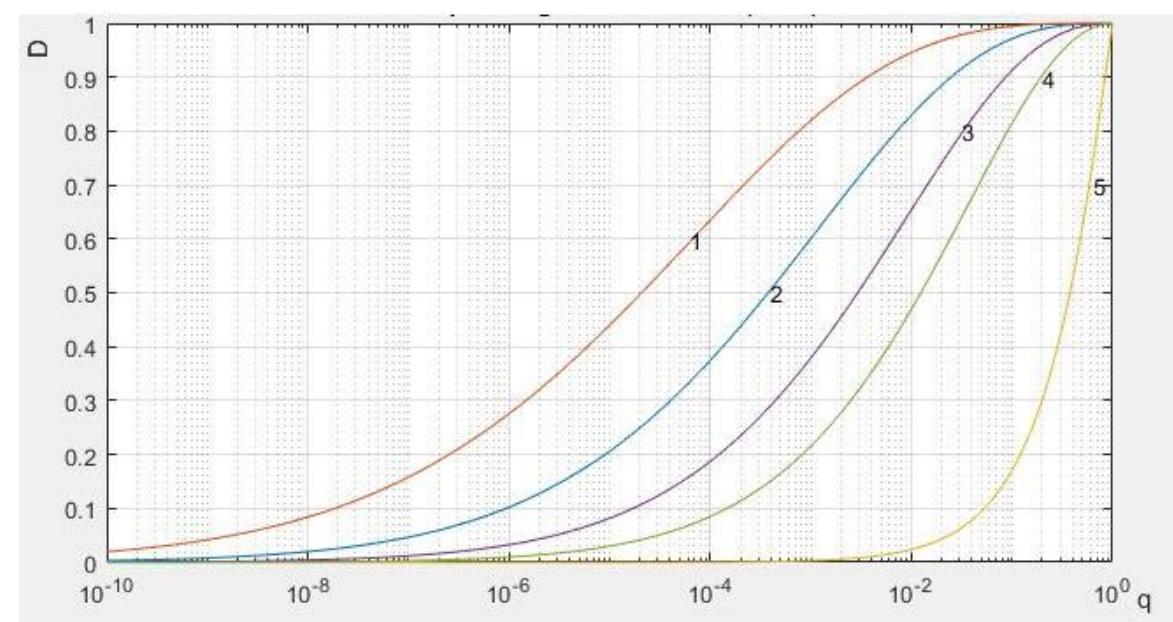

Fig. 7: Confidence of Disclosure Personality on the Integration Time

\section{Detecting the Target in Motion}

In this paper, the researcher has dealt with the detection quality in the case of an inactive object. In case, if an object is in motion, the complication arises when it passes from one resulting cell to another cell during the appearance of the pulse repetition stage [22]. This problem can be overcome by using a multi-channel device that is connected to a Doppler filtration system that organises the best revelation of moving objects. When recognising objects with varied physical lengths $\mathrm{L}$, the corresponding multichannel design can be used to choose the best integration time $\mathrm{T}$. The losses resulting from the design's multichannel structure can be measured by employing the same multi-channel digital Doppler systems' general processes.

\section{Advantages of Ultra Wideband (UWB) Radar}

The major advantages of UWB radar are large bandwidth, carrier less signal and ultra-short width of the pulse. These are explained below:

\subsection{Large Bandwidth}

The bandwidth of the UWB signal is very large so it covers a large range. This feature is good for image processing such as for high resolution of image and high rate of data transmission. High rate assists in getting the same signal through the walls and in getting images related to biomedical. The range of frequency for UWB is from 3.1 to $10.6 \mathrm{GHz}$ which assists the UWB radars in increasing the resolution of the image [23].

\subsection{Ultra-short Pulse Width}

This feature also provides a high resolution of the signal and also increases the data rate of the signal being transmitted. The signal transmitted from UWB has low power due to an ultra-short pulse. This feature helps in increasing the localizing accuracy and also reduces the effect of 
interference. Hence, these features increase the resolution of the signal [24]. This feature is mostly utilized for indoor localization.

\subsection{Carrier less Signal}

The advantage of UWB is that there is no need for a carrier signal to module the signal in case of UWB radar systems. It can modulate the signal over the high bandwidth without utilizing the carrier signal [25]. The signal is transmitted in the form of pulse which is a digital transmission, so due to this reason, the UWB radar system has a simple hardware structure. Due to its low power feature, it operates on the low range of power.

\section{Conclusion}

The main possibilities of UWB radars include advanced detected object array dimension efficiency, identification of object class and sort, reduction in the radar possessions of reflexive obstruction, enhanced cohesion observing point at low ascent edge, development of the feasibility of object detection and enhanced balance observing, slender aerial mould by changing the radiated signal quality, development of the radar's exemption to outside narrowband electromagnetic emission possessions and commotion, reduction in the radar "dead zone" and amplification of the radar's furtiveness by a gesture. The main characteristics of UWB radars are the variations in signal waveform during detection and ranging, as well as the antenna pattern's dependence on the signal duration and waveform. When used under static jamming conditions, the UWB radar has an extra feature that distinguishes it from narrow-band radars. In order to detect a target in the UWB Radar, correct picture dispensation is required, which allows for the distinction of an object and the generation of a radio image of that object. A moving object can be detected by means of a multi-channel device-related Doppler filtration system. The major advantages of UWB radar are large bandwidth, carrier less signal and ultrashort width of the pulse.

\section{Acknowledgments}

The European Union's Horizon 2020 research and innovation programme under grant agreement H2020-MSCA-ITN-2016 SECRET-722424 and the UK's Research Council (EPSRC) under grant EP/E022936 partially sponsored this work.

\section{Reference:}

1. Neekoogar, F., Ultra-Wideband Communications, NJ, 2005: Englewood Cliffs, Prentice Hall.

2. Arslan, H., Chen, Z. N., and Benedetto, M. G. D., Ultra Wideband Wireless Communication, 2006: New York, Wiley.

3. Nikookar, H., and Prasad, R., Introduction to Ultra Wideband for Wireless Communications, 2008: New York, Springer-Verlag.

4. Immoreev, I., Use of Ultra-Wideband Location in Air Defense: Questions of Special Radio Electronics, Radiolocation Engineering Series, 1991. 22: p. 76-83.

5. Immoreev, I., Ultrawideband (UWB) Radar Observation: Signal Generation, Radiation and Processing, European Conference on Synthetic Aperture Radar (EUSAR'96), Konigswinter, Germany, 1996. 
6. Skolnik, M. I., Introduction to Radar, 1962: Radar Handbook, vol. 2.

7. Richards, M. A., Scheer, J. A., and Holm, W. A., Principles of Modern Radar, 2010: Citeseer.

8. Kang, Y., Kim, K., and Scott, W. R., Modification of Sinuous Antenna Arms for UWB Radar Applications, IEEE Transactions on Antennas and Propagation, 2015. 63: p. 5229-5234.

9. Z. Ruan, Li, C., and Peng, L., Design and Analysis of Planar Antenna with Dual WLAN BandNotched for Integrated Bluetooth and UWB applications, Journal of Electromagnetic Waves and Applications, 2010, 24: p.1817-1828,

10. Bond, E. J., Xu, L., Hagness, S. C., and Veen, B. D. V., Microwave Imaging Via Space-Time Beamforming for Early Detection of Breast Cancer, IEEE Transactions on Antennas and Propagation, 2003, 51:p. 1690-1705,

11. Muqaibel, A., Safaai-Jazi, A., Bayram, A., Attiya, A., and Riad, S., Ultrawideband Throughthe-Wall Propagation, IEE Proceedings-Microwaves, Antennas and Propagation, 2005, 152: p. 581-588.

12. Sharkawy, M., Demir, V., and Elsherbeni, A. Z., Plane-wave Scattering from ThreeDimensional Multiple Objects Using the Iterative Multi-region Technique Based on the FDFD Method, IEEE transactions on antennas and propagation, 2006, 54: p. 666-673,.

13. Allen, B., Dohler, M., Okon, E., and Malik, W., Brown, A., and Edwards, D., Ultra-wideband Antennas and Propagation for Communications, Radar and Imaging, 2006, John Wiley \& Sons. 14. Zhang, G.X., Jin, W.D. and Hu, L.Z., Radar Emitter Signal Recognition Based on Complexity Features, Journal of Southwest Jiaotong University, 2004. 12(2):p.116-122.

15. Kawalec, A. and Owczarek, R., Radar Emitter Recognition Using Intrapulse Data. In 15th International Conference on Microwaves, Radar and Wireless Communications, IEEE Cat. No. 04EX824), 2004, 2: p. 435-438.

16. Immoreev, I., Ultrawideband Location: Main Features and Differences from Common Radiolocation, Electromagnetic Waves and Electronic Systems, 1997. 2(1): p. 81-88.

17. Immoreev, I., Signal Processing in Ultra Wide Band (UWB) Radars, Fifth International Conference on Radar Systems (RADAR'99), France, Brest, 1999.

18. Immoreev, I., and Taylor, J. D., Selective Target Detecting Short Pulse Ultrawideband Radar System, Euro Electromagnetic (EUROEM2000), Scotland, Edinburgh, 2000.

19. Immoreev, I., Main Features Ultra-Wideband (UWB) Radars and Differences from Common Narrowband Radars, Ultrawide Band Radar Technology, 2000: Boca Raton, London, New Work, Washington, CRC Press.

20. Immoreev, I. Features of Signals Detecting in the UWB Radars, Ultrawide Band Radar Technology, 2000: Boca Raton, London, New Work, Washington, CRC Press. 21. Immoreev, I., and Shunjun, W., Use Ultra Wideband Radar Systems for Increased Information About Target, 2001 CIE International Conference on Radar, RADAR 2001, Beijing, China, October 15- 18, 2001.

22. Batra, A., Lingam, S., and Balakrishnan, J. Multi-band OFDM: A Cognitive Radio for UWB, Proceedings of 2006 IEEE International Symposium, 2006: p. 4097. 
23. Venkatasubramanian, V., and Leung, H., A Novel Chaos-Based High-Resolution Imaging Technique and its Application to Through-the-Wall Imaging, IEEE Signal Processing Letters, 2005. 12: p. 528-531.

24. Immoreev, I. I., and Fedotov, P. D. V., Ultra-Wideband Radar Systems: Advantages and Disadvantages, Ultra Wideband Systems and Technologies, 2002: p. 201-205.

25. Palmer, J., Longstaff, I., Martorella, M., and Littleton, B., ISAR Imaging Using an Emulated Multi-Static Radar System, IEEE Transactions on Aerospace and Electronic Systems, 2005. 41: p. 1464-1472, 2005. 